\title{
L'évolution des distinctions de durée vocalique dans la flexion nominale du français
}

\author{
Yves Charles Morin \\ Université de Montréal \\ Yves.Charles.Morin@umontreal.ca
}

Dans un grand nombre de noms et adjectifs, les marques de flexion associées aux suffixes de l'ancien français $-s$ (dans les oxytons) et $-e(s)$ (dans les paroxytons) se sont transportées sur les voyelles toniques où elles se sont transmuées en distinction de durée $\mathrm{i}$. Je n'examinerai ici que celles dont les formes fléchies se terminent maintenant par une voyelle au pluriel, comme né, nés, née, nées. J'ai porté dans le tableau 1 , les trois grands schémas distributionnels qui semblent s'être développés dans les parlers gallo-romans d'oïl à partir d'un état vraisemblablement identique : le schéma classique, celui du Nord-Ouest et celui de l'Est. Ces appellations géographiques reflètent seulement la localisation des quelques parlers examinés, non un inventaire systématique des données disponibles. Elles doivent être comprises comme des termes suggestifs en quête de précision. Le quatrième schéma, celui du Nord-Est (essentiellement le domaine wallon), bien que très voisin de celui de l'Est, semble s'être développé à partir d'un système prosodique pour la durée vocalique fondamentalement différent des autres régions et ne sera pas examiné ici (cf. Morin 2003). Il ne sera pas question non plus du ou des schémas de type picards. Ces parlers, dans leur forme stéréotypique, ne connaissent plus depuis longtemps, s'ils l'ont jamais connu, le système prosodique de durée vocalique des autres parlers gallo-romans d'oïl caractérisé par la présence de voyelles longues issues de l'affaiblissement des $s$ préconsonantiques, comme dans FĔSTĂM $>$ afr. feste

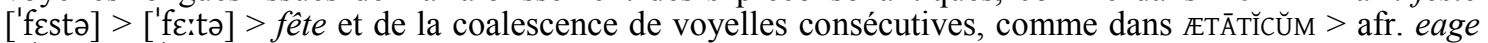
[e'adzə] > ['a:dzə] > âge (cf. Morin \& Dagenais 1988).

\begin{tabular}{|c|c|c|c|c|c|c|}
\hline & $\begin{array}{c}\text { NĀTŬM, } \\
\text { FĪNĪTǓM, } \\
\text { cō(N)sūTŭM }\end{array}$ & $\begin{array}{c}\text { NĀTŌS, } \\
\text { FĪNĪTōs, } \\
\text { cō(N)sūTōs }\end{array}$ & $\begin{array}{l}\text {-ĬTTŌs, } \\
\text {-ŎTTŌs }\end{array}$ & $\begin{array}{l}{ }^{\circ} \text { MĪSŬM, } \\
\text { RĚCLŪSǓM }\end{array}$ & NĀSŬM & $\begin{array}{c}\text { NĀTA(s), } \\
\text { FīNīTA(s), } \\
\text { cō(N)SŪTA(s) }\end{array}$ \\
\hline & $\begin{array}{l}\text { né, fini, } \\
\text { cousu }\end{array}$ & $\begin{array}{l}\text { nés, finis, } \\
\text { cousus }\end{array}$ & sifflets, sots & mis, reclus & nez & $\begin{array}{l}\text { née(s), finie(s), } \\
\text { cousue(s) }\end{array}$ \\
\hline Classique & {$[\mathrm{e}, \mathrm{i}, \mathrm{y}]$} & \multicolumn{5}{|c|}{$[\mathrm{e}: \mathrm{i}: \mathrm{y:}]-\left[\varepsilon^{\prime}, \mathrm{o:}\right]$} \\
\hline Nord-Ouest & \multicolumn{2}{|c|}{$[e, \mathrm{i}, \mathrm{y}]$} & \multicolumn{4}{|c|}{$[\mathrm{e}: \mathrm{i}:, \mathrm{y:}]-\left[\varepsilon^{\prime}, \mathrm{o:}\right]$} \\
\hline Est & \multicolumn{4}{|c|}{$[\mathrm{e}, \mathrm{i}, \mathrm{y}]-[\mathrm{o} / \mathrm{a} / \propto]$} & \multicolumn{2}{|r|}{$[\mathrm{e}: \mathrm{i}:, \mathrm{y}:]$} \\
\hline Nord-Est & \multicolumn{5}{|c|}{$[\mathrm{e}, \mathrm{i}, \mathrm{u} / \mathrm{y}]-[\varepsilon, \mathrm{o}]$} & [e:i , i:i , uu/ui /yu/ \\
\hline
\end{tabular}

Tableau 1. Schéma général d'évolution des marques de flexion $-s$ et -e

Les timbres vocaliques indiqués ici sont simplement indicatifs et illustrent des grandes classes de sons dont les valeurs précises peuvent être relativement différentes dans différents dialectes et qui ont beaucoup évolué dans le temps. Pour les parlers de l'Est, [ə/a/œ] note les reflets de [e] et [o] romans dans [-etos] < -ĬTTōs et [-otos] <-ŏтTōs. Les reflets de -ĀSǓM (terminaison rare en dehors de NĀSǓM, RĀSǓM) sont peu représentés dans le lexique et mal documentés dans les documents examinés ; leur classement est ici tout sauf définitif.

Le schéma classique, normalement admis pour la langue standard, apparaît particulièrement simple. Les voyelles toniques y résulteraient d'un allongement compensatoire dû à l'affaiblissement d'un $s$ final pour le masculin pluriel et de la fusion avec un chva posttonique pour le féminin. L'évolution comparée des autres parlers montre qu'on doit probablement distinguer deux étapes pour la perte du $-s$ final. Une ancienne, en sandhi devant un mot commençant par une consonne, avec allongement compensatoire de la voyelle précédente, et une plus récente, à la pause, n'ayant pas eu d'effet sur la durée de cette voyelle.

Le schéma du Nord-Ouest s'observe en gallo (Bretagne romane), pour lequel on dispose d'une excellente analyse (Chauveau 1984: 159-169, 1989: 16-39), et qui vaut très certainement pour un domaine beaucoup plus vaste. Chauveau argumente contre une analyse simplement morphologique qui voudrait que le schéma du Nord-Ouest constitue une étape plus évoluée du schéma classique après que les formes du masculin pluriel comme nés, finis, cousus - originalement avec des voyelles toniques longues aient été refaites sur celles du singulier, permettant à la durée (ou aux différences de timbres qui en sont issues) d'opposer les formes du masculin (sans distinction de nombre) aux formes du féminin. L'argument repose sur les propriétés spécifiques de l'affriquée $[\mathrm{ts}]<[\mathrm{t}]+[\mathrm{s}]$ résultant de l'évolution 
phonétique régulière des terminaisons -ĀTōs, -īTōs, -ūTōs $>$ [-a:t(o)s, -i:t(o)s, -u:t(o)s] $>$ [-ett+s, -it+s, -yt+s] $>$ [-ets, -its, -yts], qui n'ont pas eu les mêmes effets que le [s] primitif sur la voyelle précédente.

Les schémas de l'Est et du Nord-Est sont mentionnés ici pour mémoire. Le premier repose sur une analyse en cours du parler bourguignon de Bourberain (Rabiet 1887-1890). Il n'en sera plus question dans la suite de cette présentation"

Dans le reste de ce travail, j'examinerai les thèses qui ont été proposées sur le développement de la durée vocalique des toniques dans les oxytons et comment ces thèses rendent compte des différences enregistrées par les grammairiens du XVI ${ }^{\mathrm{e}}$ siècle (j'entends, ceux dont le témoignage est suffisamment riche). Partant de cette étape, probablement partagée par les parlers ayant adopté le schéma du NordOuest et le schéma classique, j'examinerai les traces qu'on peut relever de leur développement jusqu'à l'époque moderne, dans le parler de Pléchâtel (Dottin et Langouët 1901) pour le schéma du Nord-Ouest et dans la norme parisienne décrite par l'abbé Rousselot (Rousselot et Laclotte 1913) pour le schéma classique.

\title{
1 Les sources de la durée oxytonique
}

Selon l'opinio communis, la durée des toniques des pluriels des substantifs oxytoniques résulterait d'un allongement «compensatoire» provoqué par la chute du $s$ de flexion en finale de mot, changement semblable à celui que l'on connaît pour les $s$ préconsonantiques à l'intérieur des mots, comme dans fête < feste.

\subsection{Fouché (1958-1961 / 1966-1969)}

C'est essentiellement cette thèse qu'adopte Fouché $(1966,1969)$, qui essaie cependant d'accomoder les témoignages discordants des premiers grammairiens. Les idées du maître sont difficiles à suivre, non seulement parce qu'il lui arrive de présenter dans différentes parties de son manuel des analyses qui semblent contradictoires ${ }^{\text {iii }}$, mais surtout parce sa présentation des témoignages anciens est succincte, tronquée et très sélective - se limitant apparemment à ceux qui lui semblent compatibles avec l'évolution générale de la langue telle qu'il la conçoit.

Les données non conformes à la thèse de l'allongement compensatoire seraient le produit d'analogies morphologiques les plus diverses. C'est ainsi que, là où la tonique est brève au pluriel, elle aurait été refaite sur le singulier :

\begin{abstract}
Il ne faudrait pas croire que la règle de l'allongement vocalique devant un $s$ final non prononcé n'ait pas souffert d'exceptions chez les premiers grammairiens. Ainsi pour Péletier (1549), les $2^{\mathrm{e}}$ pers. plur. donnez, allez ont une voyelle accentuée brève, de même que les participes passés pluriels preparez, ebahiz, nourriz, suz, vuz, etc. Sans doute, dans ce dernier cas, faut-il recourir à l'action analogique des formes correspondantes du masculin singulier. A leur tour, les participes donnez, allez ont pu influencer les $2^{\mathrm{e}}$ pers. plur. de l'indic. prés. (Fouché 1969: 390)
\end{abstract}

Le témoignage de Peletier est cependant insuffisant pour comprendre la nature des changements qui se sont produits. Celui-ci ne note les durées que dans un nombre relativement limité de cas, essentiellement lorsqu'elles résultent de la chute d'un $s$ préconsonantique - pour contrer les arguments des défenseurs de l'orthographe ordinaire contre ses propositions de réforme - et dans les terminaisons des formes verbales où elles jouent un rôle distinctif dans la morphologie (cf. Morin 2004). Le dictionnaire des rimes de Lanoue (1596) est beaucoup plus riche et enregistre un grand nombre d'autres voyelles longues incompatibles avec la thèse de l'allongement compensatoire, qui ne peuvent cependant pas toutes s'expliquer par un recours - même très libéral - à ce genre d'analogie. C'est ainsi, par exemple, que bras, materas, assez, brebis, dix, perdrix, tapis et vis ont une tonique brève, aussi bien au singulier qu'au pluriel, sans modèle analogique possible permettant d'expliquer la perte d'une durée qui aurait été produite par l'effacement d'un ancien $-s$ final.

Dans l'analyse de Fouché, la perte du $s$ en finale de mot est un changement phonétique régulier (1966: 678) qui s'était produit dans la langue ordinaire pendant la « $2^{\mathrm{e}}$ moitié du XII $\mathrm{XI}^{\mathrm{e}}$ siècle et le XIII" ${ }^{\mathrm{e}}$ siècle " (p. 663), mais beaucoup plus tard dans la langue savante puisque : «En 1549, R. Estienne écrit que l's "se prononce partout où l'on s'arreste" » (p. 666). Ceci laisse en suspend une question fondamentale. Si c'est l'effacement phonétique du $s$ final qui provoque l'allongement d'une tonique précédente, comment la durée s'est-elle développée dans la langue des grammairiens, qui a conservé cette consonne finale à la 
pause ? Contrairement à ce que laisse entendre Fouché, les graphies réformées de Peletier qu'il cite ne notent pas la durée des voyelles « devant un $s$ final non prononcé », mais celles qu'elles ont dans la forme de citation des mots, où au contraire le $s$ final était prononcé ${ }^{\text {iv }}$.

\title{
1.2 Pope (1934 / 1952)
}

Pope (1952) avait bien vu ce problème et avait tenté d'y apporter une solution. Son analyse distingue deux étapes pour l'évolution des mots se terminant par $-s$. Comme les autres obstruantes en finale de mot, cette consonne se serait d'abord effacée en sandhi devant consonne, où sa perte aurait produit le même allongement compensatoire qu'à l'intérieur des mots :

\begin{abstract}
Effacement of final consonants varied in date according to the nature of the consonants and its position in the word. The first to be affected are final $s(z)$, a consonant that was in process of effacement in prae-consonantal position in the interior of a word (§ 377) and final supported plosive consonants, [...] Examples of effacement of these consonants in prae-consonantal position appear in [AngloNorman] mss. of the later twelfth century. (Pope $1952: 220, \S 613$ )
\end{abstract}

Les alternances produites par les divers sandhis externes de la langue médiévale ont cependant eu tendance à se simplifier par la généralisation d'une des variantes à d'autres positions que celle où elle avait pris naissance. Ce genre de généralisation peut se produire très tôt pour certains mots dans certains usages ou pour certains registres, mais ce n'est qu'au cours du XVII ${ }^{\mathrm{e}}$ siècle que la norme enregistre la généralisation à la pause des formes qui s'étaient développées ailleurs devant consonne :

So varying a pronunciation of the final consonants of words imposed a heavy burden on the memory and people soon began to make things easier for themselves by reducing the number of forms employed. The earliest and commonest method adopted was to use at the pause the prae-consonantal form, the one with effaced final consonant. (Pope 1952: 221, § 614)

$\mathrm{Au} \mathrm{XVI} \mathrm{X}^{\mathrm{e}}$ siècle, cependant, les variantes prépausales primitives avec un $-s$ final, flexionnel ou non, pouvaient toujours s'entendre, comme Pope le relève dans les témoignages de Meigret (1548-1551), Peletier (1550-1580) et Lanoue (1596). Or leurs voyelles toniques peuvent être longues, ce qui ne saurait résulter de l'effacement du $s$ final. Pope y voit l'effet d'une autre forme d'allongement compensatoire, provoqué cette fois par la perte d'une consonne précédant le $-s$ final :

It is probable also that the Old French effacement of the final consonants before flexional $[\mathrm{s}]$ had produced a lengthening that was still perceptible in the sixteenth century, for Meigret distinguishes between the length of vowels in the singular and plural of such words as end in the singular in a consonant that was mute in the plural $^{v}$, e.g. between lac : lacs [la:s], coc : cocs [ko:s], hanap : hanaps [hana:s] and Peletier and Lanoue distinguish between the length of the vowel in the plural of words of this type and in the plural in which there was no such effaced consonant. (Pope 1952: $207, \S 567)$.

Cette thèse de Pope est peu plausible. La dernière proposition est relativement sibylline et constitue peutêtre un aveu d'impuissance à régler le problème. Elle y fait probablement allusion aux formes du type ami [ami], pl. amis [amiss] relevées par Lanoue, où l'on peut difficilement concevoir que la durée soit compensatoire. Inversement, la perte d'un $-t$ devant $-s$, comme dans fagot [fagot], pl. fagots [fagos], ne s'accompagne jamais de l'allongement de la voyelle précédente (sauf pour petit) — un cas véritablement exceptionnel qui ne peut provenir que d'un développement particulier.

On retiendra cependant de la proposition de Pope que la forme moderne des mots à la pause peut avoir des sources multiples, correspondant à des évolutions phonétiques régulières, soit à la pause, soit ailleurs devant consonne. On ne peut exclure que le $s$ en final de mot se soit effacé à deux périodes différentes, une première fois devant consonne pendant la période médiévale et une seconde fois à la finale absolue, probablement à la fin du XVI et au début du XVII siècle dans la langue des lettrés, ce second effacement, également phonétique, n'ayant cependant pas été accompagné d'allongement compensatoire.

Cette hypothèse n'exclut pas que les formes préconsonantiques puissent aussi avoir été généralisées à la pause selon le modèle général des sandhis consonantiques du français (cf. Morin 2005a). Il y aurait donc eu deux sources pour l'effacement du [s] dans les formes du pluriel de la langue moderne : un effacement phonétique ancien devant consonne, avec allongement compensatoire, et un effacement phonétique plus récent à la pause, sans allongement compensatoire. 


\section{La situation au $\mathrm{XVI}^{\mathrm{e}}$ siècle}

\subsection{Lanoue (1596)}

Le tableau 2 donne les paradigmes du nombre pour les substantifs et les adjectifs oxytoniques dont la tonique est immédiatement suivie de $s$, tels qu'ils apparaissent dans le dictionnaire de rimes de Lanoue (cf. Morin et Desaulniers 1991 pour un aperçu des autres paradigmes et leur classement) ${ }^{\mathrm{vi}}$. L'auteur note les prononciations des mots à la rime, donc à la pause. J'ai indiqué sous la colonne « $-\mathrm{C} »$, la prononciation correspondante la plus probable de leur terminaison devant un mot commençant par une consonne.

Les paradigmes $\mathrm{A}$ et $\mathrm{C}$ regroupent les noms dont les toniques ont la même durée pour les deux nombres : tonique brève pour $\mathrm{A}$ et tonique longue pour $\mathrm{C}$. Les paradigmes $\mathrm{B}$ regroupent ceux dont la tonique est brève au singulier et longue au pluriel.

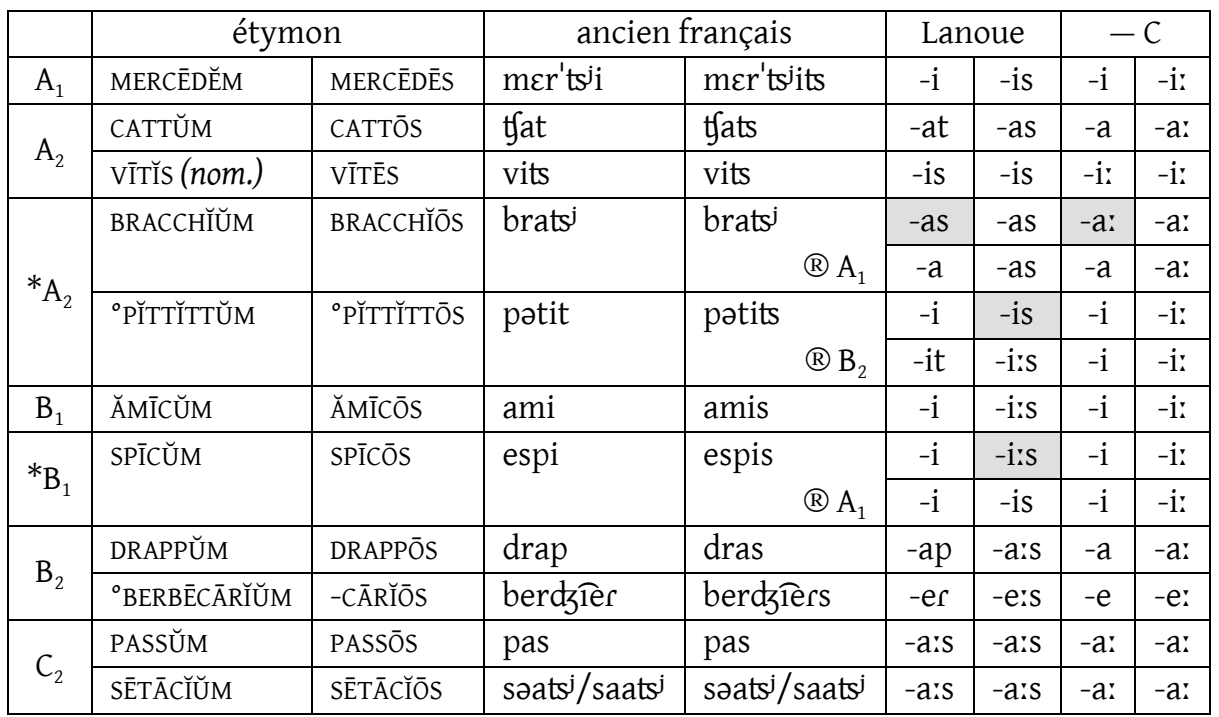

Tableau 2. Les paradigmes du nombre dans le dictionnaire de Lanoue (1596)

Le tableau 2 illustre aussi les changements analogiques observés, pour lesquels le paradigme attendu apparaît dans la colonne de gauche précédé d'un astérisque tandis que le paradigme analogique observé suit le signe « ${ }^{\circledR} »$. Par exemple, «* $\mathrm{A}_{2}{ }^{\circledR} \mathrm{A}_{1}$ » illustre un changement où l'on aurait attendu le paradigme $A_{2}$ mais où le résultat a été le paradigme $A_{1}$; la forme phonétique régulière qui a été remplacée apparaît en grisé.

L'évolution des toniques à la pause est remarquable. Sont longues celles qui étaient suivies de [s] ou [rs] en ancien français, comme dans amis et dras (pl. de drap). Sont brèves celles qui étaient suivies des affriquées [ts'] et [ts] comme dans merciz (pl. de merci) et braz 'bras', à moins qu'elles ne se soient combinées à une voyelle précédente, comme dans saaz 'sas'. Les exceptions, du type épi ou petit notés dans ce tableau, sont extrêmement rares ${ }^{\text {vii }}$.

\begin{tabular}{|c|c|c|c|}
\hline étymon & a.fr. & Lanoue & \\
\hline 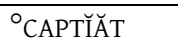 & ['tfats'a] & ['Jasə] & (il) chasse \\
\hline${ }^{\circ}$ CŏČ̆ $\breve{A M}$ & ['kots'ə] & ['kosə] & cosse \\
\hline DĪRĒCTǏĂT & ['dretsja] & ['dresə] & (il) dresse \\
\hline${ }^{\circ}$ MŪCǏ̃̆T & ['myts'a] & ['mysə] & (il) musse \\
\hline PĔLLǏČĬAM & [pə'litsjə] & [рә'lisə] & pelisse \\
\hline${ }^{\circ}$ CORRŬPTǏĂT & [kor'rots $\left.{ }^{\mathrm{j}} \partial\right]$ & [ku:'rusə] & (il) courrouce \\
\hline
\end{tabular}

\begin{tabular}{|c|c|c|c|}
\hline étymon & a.fr. & Lanoue & \\
\hline CAPSĂM & ['tfasə] & ['Ja:sə] & châsse \\
\hline FŎSSĂM & ['fosə] & ['fo:sə] & fosse \\
\hline PRĔSSĂT & ['presə] & ['pre:sə] & (il) presse \\
\hline FǓǏSSĔM & ['fyso] & ['fy:sə] & (je) fusse \\
\hline FĪNī(v)ǏsSĚM & [fə'nisə] & [fi'ni:sə] & (je) finisse (subj.) \\
\hline RǓSSĂM & ['rosa] & ['ru:sə] & rousse \\
\hline
\end{tabular}

Tableau 3. Allongement des voyelles devant $s$ dans les paroxytons 
L'allongement des toniques des oxytons devant $[\mathrm{s}]$ et [ts/t $\left.\mathrm{s}^{\mathrm{j}}\right]$ en finale de mot est entièrement parallèle à celui qu'on observe devant [sə] et [tsja] dans les paroxytons, comme on peut voir dans le tableau 3 . Il fait peu de doute que l'allongement a les mêmes causes dans les oxytons et dans les paroxytons.

Le mécanisme responsable de cet allongement n'a pas encore reçu d'explication satisfaisante. On a voulu y voir un effet de la dégémination du [ss] roman (Dumas 1986: 177), ce qui exige cependant que ce changement se soit produit « pendant la période moyenne de la langue », comme le proposait encore en 1958, Édouard Bourciez dans son manuel (cf. § 155 Rem. II). Cette date a été abandonnée dans la révision que fait Jean Bourciez du même manuel en 1967. De plus cette explication ne vaudrait que pour les paroxytons. Une autre explication, plus ingénieuse que convaincante, a été proposée par Joos (1952) et reprise par Martinet (1955: 245-46).

On ne peut exclure que l'allongement ne tire sa source de différences de durée à l'origine relativement secondaires devant [s] et [ts], qui se seraient amplifiées pour compenser la perte des distinctions entre la fricative et l'affriquée correspondante selon le schéma $[-\operatorname{as}(\partial)] \neq\left[-{ }^{\prime}\right.$ ăts $\left.(\partial)\right]>\left[-{ }^{\prime} \operatorname{ars}(\partial)\right] \neq[-\operatorname{as}(\partial)]$. Il est révélateur que cet allongement affecte les voyelles toniques (d'où elle peut s'étendre à d'autres positions dans les dérivés), mais non les prétoniques, comme le a de assez et de assis. L'allongement semble donc relever de l'organisation prosodique de la langue, ce qui exclut l'analyse de Joos dans laquelle il proviendrait de différences articulatoires entre [s] et [ts], et devrait donc être indépendant de l'accentuation des voyelles qui les précèdent.

\subsection{Peletier (1550-1581)}

Le témoignage de Peletier est beaucoup moins riche que celui de Lanoue, nous l'avons vu. Dans ses premiers textes en orthographe réformée (1550-1555), la distribution des durées des pluriels est entièrement conforme à celle que donne Lanoue. En particulier, les pluriels de participes passés issus de -ĀTŌS, -ĪTŌS et -ŪTōS du paradigme $\mathrm{A}_{1}$ sont régulièrement notés avec une tonique brève : acusèz, nèz (part. passé de naître), dфpartìz, randùz.

Dans sa dernière œuvre en orthographe réformée de 1581, cependant, ceux-ci apparaissent avec une voyelle longue : acuséz, néz, departíz, randúz (cf. Morin 2005b). Le grammairien manceau avait-il définitivement changé de norme de prononciation? Est-ce l'indice que l'on commence à généraliser les variantes préconsonantiques du paradigme $\mathrm{A}_{1}$ dont les voyelles toniques étaient longues ? Rien dans l' "Avęrticømant aus Lecteurs" ne permet de comprendre ce qui a motivé l'auteur à noter ces changements.

\subsection{Meigret (1548-1551)}

La langue de Meigret a de nombreux traits particuliers, qui correspondent probablement à des usages régionaux du français de Lyon (cf. Shipman 1953). Un de ceux-ci est le timbre [e] de la voyelle posttonique. Meigret distingue ainsi la forme verbale de la $3 \mathrm{~s}$ du présent de l'indicatif (il) eyme ['cime] du participe passé eymé [عi'me] par la position de l'accent tonique seulement. Dans son cadre théorique, cette différence ne peut être qu'une différence de durée, ce qui est objectivement le cas, la tonique étant plus longue que la posttonique. Cette analyse est possible parce que, comme dans la langue de Lanoue et dans celle de Peletier pendant la première période, il n'y a pas de différence de durée entre les toniques du participe passé au singulier et au pluriel, ce qui lui fait dire que les deux toniques sont également longues. Il n'y avait apparemment pas de différences non plus dans son usage entre le nom nés 'nez' et le participe passé nés de naître. Contrairement à Lanoue et à Peletier pendant sa première période, cependant, Meigret distingue les durées des toniques issues de -ĪTŬM et de -ĪTōs: bany $\neq$ banís, comme celles de -ŪTŬM et de -ŪTōs : tenu $\neq$ tenús.

\section{Le système du Nord-Ouest}

Ce n'est qu'à partir du moment où des formes du pluriel sans $-s$ final appaissent à la pause, que l'on peut voir apparaître les différents systèmes du tableau 1. Je dirai qu'un usage préfigure ou anticipe le système du Nord-Ouest quand, minimalement, les reflets des terminaisons -ĀTōS sont des voyelles brèves dans une variété de langue qui connaît des distinctions de durée à la pause (ou des différences de timbre qui proviennent de telles distinctions). Même avec cette définition très libérale, trois grammairiens seulement illustrent ce système. 
Il s'agit d'abord de La Touche, qui pourrait être originaire des pays de la Loire. Il recommande à ses lecteurs de prendre des leçons auprès de maîtres ayant «fait dès leur jeunesse un long séjour dans les lieux où l'on parle bien, comme à Paris, à Saumur où [sic] à Blois, qui sont les villes dont l'accent aproche le plus celui de la Cour » (1696, fin de l'introduction). Le second, J. B. Roche a fait imprimer son livre à Nantes et le dédie à la Marquise de Coutance, certainement dame Anne-Blanche-Victoire Cauchon de Maurepas, dame de la Haie-Mahéas, de Cordemais, etc. On ne sait rien des origines de Vaudelin, le troisième.

\subsection{La Touche (1696-1730)}

Un siècle après le dictionnaire des rimes de Lanoue, Pierre de La Touche publie la première édition de son L'art de bien parler françois, dont les observations sur la prononciation semblent surtout inspirées par son usage personnel et relativement indépendantes du discours dominant.

Comme c'est normalement le cas du témoignage des traités grammaticaux, le sien est relativement pauvre en comparaison des données rassemblées dans les dictionnaires ${ }^{\text {viii }}$. Le traité de La Touche permet cependant de voir une généralisation importante de la durée comme marque du pluriel des oxytons. Tous les noms et adjectifs dont la tonique brève est suivie de [t] au singulier perdent cette consonne et allongent la tonique au pluriel : faits (=fầ, p. 10), soldats (=soldâ, p. 10), fagôts (=fagô, pp. 23, 25, 44), états $(=e ́ t a ̂$, p. 25), habits, tributs (p. 44). Par contre, les oxytons qui se terminaient par une voyelle à l'époque médiévale, ont au pluriel la durée que leur attribuait Lanoue. Ont ainsi une voyelle brève au pluriel : les participes passés issus de -ĀTōs, -ĪTōs et -ŪTōs : abrégés, aimés, finis, reçus (p. 43) et l'adjectif jolis. Ont une voyelle longue : le pluriel amis < AMĪCōs de ami (p. 45) et le nom nez (p. 47) < NASŬM, NASŌS pour les deux nombres.

L'édition de 1730 du même ouvrage donne des indications beaucoup plus riches sur la prononciation et contient cette fois de nombreuses critiques de descriptions d'autres grammairiens. On y retrouve les mêmes observations sur l'allongement au pluriel des oxytons se terminant par [t] au singulier. Étrangement, cependant, la section consacrée à l'examen des durées vocaliques des oxytons (1696:4447) a été supprimée. L'auteur justifiait ainsi la partie consacrée à la prosodie dans sa première édition : «Comme la plus grande \& prefque la feule dificulté confifte dans les fylabes finales, \& fur tout dans les pénultièmes, je ne parlerai point des autres » (1696: 43). Ce qu'il a révisé ainsi ultérieurement: "Comme la plus grande, \& prefque la feule dificulté, à l'égard de la Langue Françoife, confifte dans les pénultièmes fylabes, je ne parlerai point des autres » (1730: 68). C'est un signe assez sûr de l'existence d'une forte variation dans l'usage à cette époque, dont l'auteur — émigré en Angleterre - a fini par prendre conscience et dont il a, par prudence, préféré ne plus parler.

\subsection{Vaudelin (1713-1715)}

Dans deux ouvrages écrits probablement au cours des années 1680 (cf. Walter 1993: 211), où il met en application sa propre orthographe réformée, Vaudelin note souvent longue la tonique des oxytons qui se terminaient par [t] dans les mots suivants : contrat, magistrat, esprit (1occ./2), profit, alphabet, parfait, secret, mot (4 occ./14). Contrairement à l'usage de la Touche, cependant, ces mots se prononcent sans [t] final.

Le [i] final du pluriel de ami, avis, banni, commis, ennemi, mari, mercredi, promis, ravi, rempli, samedi, vendredi n'est jamais long, ni le [y] final du pluriel de conçu, corrompu, défendu, parvenu, superflu, ni

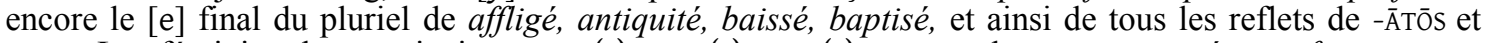
-ĀTĒs. Les féminins des terminaisons -ĨTA(S), -ŪTA(s), -ĀTA(s) comme dans garnie, guérie, infinie, partie, unie, vie, due, interrompue, reçue, venue, vue, abrégée, année, appelée ou attachée ont par contre la voyelle longue attendue au féminin.

\subsection{Roche (1777)}

Lorsqu'il écrit son traité en 1777 , J. B. Roche ne peut pas ne pas être influencé par la norme dominante de son époque qui valorise le système classique. Il est difficile de distinguer dans ses propos ce qui relève du respect des autorités de ce qui lui est propre et où il a "ofé [s']écarter de [s]es modèles » (préface, p. 2). Je mentionnerai seulement ici la triple distinction de durée qu'il fait (p. 105) entre le «E aigu bref » des reflets de -ĀTĔM dans bonté, sévérité, le «E aigu un peu alongé » des reflets de -ĀTĒS dans bontés, libertés, et enfin le «E aigu plus alongé » des reflets de -ĀTA(S) dans année, allées, ainsi que de la désinence de $2 \mathrm{pl}$ 
$-e z<-\bar{A} T \breve{s}$ (qui est généralement un [e:] long dans les parlers gallos, ou qui a le timbre caractéristique des anciens [e:] longs, comme nous verrons). On peut penser qu'il n'y avait pas vraiment de différence entre le «E aigu bref» et le «E aigu un peu alongé » dans son parler, mais qu'il s'est senti obligé de mentionner une différence que faisaient tous les grammairiens ${ }^{\text {ix }}$.

\section{Le parler gallo de Pléchâtel}

Les anciennes distinctions de durée se sont accompagnées d'importantes différences de timbre dans l'ensemble des parlers gallos — qui sont bien documentées (cf. Chauveau 1984, 1989). En règle générale, les transcriptions de Dottin et Langouët (1901) pour le gallo de Pléchâtel enregistrent seulement les différences de timbres $[ə] \neq[\mathrm{e}],[œ] \neq[\varnothing],[0] \neq[0]$ pour les anciennes oppositions de durée des voyelles moyennes $[\mathrm{e}] \neq[\mathrm{e}:],[\varnothing] \neq[\varnothing:],[\mathrm{\jmath}] \neq[\mathrm{o:}]$, mais conservent la distinction de durée sans différence de timbre pour $[\varepsilon] \neq[\varepsilon:]$. Pour les voyelles basses, ils notent à la fois des différences de timbre et de durée : [a] $\neq\left[a_{:}\right]$. Enfin, l'ancien [i:] est parfois noté long, mais le plus souvent comme une diphtongue [i:i ] ou [ii], tandis que la durée seule est distinctive pour les autres voyelles hautes: $[\mathrm{y}] \neq[\mathrm{y}:],[\mathrm{u}] \neq[\mathrm{u}:]$.

\begin{tabular}{|c|c|c|c|c|}
\hline & \multicolumn{2}{|c|}{ masculin } & féminin & f. française \\
\hline $\mathrm{A}_{1}$ & $\begin{array}{l}\text { kurbo } \\
\text { fini } \\
\text { ny }\end{array}$ & $\begin{array}{l}\text { kurbə } \\
{ }^{\circ} \text { fini } \\
{ }^{\circ} \text { ny }\end{array}$ & $\begin{array}{c}\text { kurbe } \\
\text { finii } \\
\text { ny: }\end{array}$ & $\begin{array}{l}\text { courbé } \\
\text { fini } \\
n u\end{array}$ \\
\hline $\mathrm{A}_{2}$ & $\begin{array}{l}\text { pule } \\
\text { so } \\
\text { lo } \\
\text { fago } \\
\text { pti } \\
\text { ?(bra) }\end{array}$ & $\begin{array}{l}\text { pule: } \\
\text { so } \\
\text { lo } \\
\text { fago } \\
? \\
?\end{array}$ & $\begin{array}{l}\text { pulet } \\
\text { sot }\end{array}$ & $\begin{array}{l}\text { poulet } \\
\text { sot } \\
\text { lot } \\
\text { fagot } \\
\text { petit } \\
\text { bras }\end{array}$ \\
\hline $\mathrm{B}_{1}$ & $\begin{array}{l}\text { ? (ami) } \\
\text { ? (epi) }\end{array}$ & $\begin{array}{l}? \\
?\end{array}$ & $?$ & $\begin{array}{l}\text { ami } \\
\text { épi }\end{array}$ \\
\hline $\mathrm{B}_{2}$ & $\begin{array}{l}\text { ko } \\
\text { bœe } \\
\text { bulãzə } \\
\text { prəmîn }\end{array}$ & $\begin{array}{l}\text { ko } \\
\text { bø } \\
\text { bulãze } \\
\text { prami e }\end{array}$ & $\begin{array}{l}{ }^{\circ} \text { bulãzər } \\
{ }^{\circ} \text { pramiar }\end{array}$ & $\begin{array}{l}\text { coq } \\
\text { bøuf } \\
\text { boulanger } \\
\text { premier }\end{array}$ \\
\hline $\mathrm{C}_{2}$ & $\begin{array}{l}\text { grii } \\
\text { grø } \\
\text { ne } \\
\text { sa: } \\
\text { la: }\left(<A_{2}\right)\end{array}$ & $\begin{array}{l}\text { grii } \\
\text { grø } \\
\text { ne } \\
\text { sa: } \\
\text { la: }\end{array}$ & $\begin{array}{l}{ }^{\circ} \text { gri:z } \\
\text { grøs }\end{array}$ & $\begin{array}{l}\text { gris } \\
\text { gros } \\
\text { nez } \\
\text { sas } \\
\text { lacs }\end{array}$ \\
\hline
\end{tabular}

Tableau 4. Les alternances du nombre dans le gallo de Pléchâtel (les formes précédées de ${ }^{\circ}$ ont été construites à partir des régularités décrites par les auteurs ${ }^{\mathrm{x}}$ ).

Ces parlers ont conservé un riche système de distinctions morphologiques du nombre portées directement par les noms et adjectifs. Ils ont conservé les distinctions de nombre marquées par la vocalisation du $l$

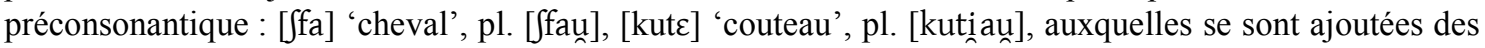
alternances du même type amenées par la simplification des diphtongues brèves: [bala] 'balai', pl. [balai ], [na] 'noir', pl. [nai ].

Le tableau 4 donne les homologues en gallo moderne des paradigmes du XVI ${ }^{\mathrm{e}}$ siècle et, lorsqu'elles existent, les formes féminines correspondantes. Le corpus ne permet malheureusement pas d'illustrer l'évolution du paradigme $\mathrm{B}_{1}$, qui correspond aux cas où la forme du singulier a une voyelle brève à la finale absolue et à laquelle correspond une longue au pluriel, comme ami, pl. amis. Il ne comprend pas non plus de continuateurs du paradigme $\mathrm{A}_{2}$ ayant un $s$ final issu du [ts] médiéval, comme vis, pis $(<$ 
PĔCTŬs) $)^{\mathrm{xi}}$; dans la discussion qui suit, le terme «paradigme $\mathrm{A}_{2}$ » doit être compris comme «paradigme $\mathrm{A}_{2}$ limité aux mots dont le singulier se terminait par $[\mathrm{t}]$ en ancien français ».

Dans les paradigmes attestés, à l'exception de $A_{1}$, la tonique du pluriel est toujours longue, que celle du singulier soit brève comme pour $A_{2}$ et $B_{2}$, ou longue comme pour $C_{2}$. Les masculins du paradigme $A_{1}$ sont invariables, avec une tonique brève autant au singulier qu'au pluriel.

Cette évolution correspond dans ses grandes lignes à celle que décrivait La Touche et Vaudelin à la fin du $\mathrm{XVII}^{\mathrm{e}}$ siècle, si l'on se permet d'extrapoler à partir de leur corpus relativement réduits. L'inconnue reste l'homologie avec le paradigme $B_{1}$ qui divergeait chez La Touche et Vaudelin, le premier ayant une tonique longue au pluriel et le second une brève.

L'évolution des paradigmes $B_{2}$ et $C_{2}$ est phonétiquement régulière et ne soulève aucun problème. Pour ce qui est du paradigme $\mathrm{A}_{2}$, illustré par poulet/poulets, où la tonique était brève à la pause pour les deux nombres, on peut rendre compte de la longueur au pluriel de la tonique du gallo moderne de deux manières : soit que le pluriel reflète la forme préconsonantique $\mathrm{du} \mathrm{XVI}^{\mathrm{e}}$ siècle, dont la tonique était longue, soit qu'il résulte d'un alignement morphologique sur le paradigme $\mathrm{B}_{2}$, où la durée est phonétique tant à la pause qu'en sandhi devant consonne.

Le principe d'uniformité des changements historiques fait attendre la même évolution pour le pluriel du paradigme $A_{1}$ que pour celui du paradigme $A_{2}$. Le premier avait les mêmes caractéristiques générales que le second et n'en diffèrait que par la terminaison au singulier dont la tonique est à la finale absolue pour $\mathrm{A}_{1}$, et suivie de la consonne [ $\mathrm{t}$ ] pour $\mathrm{A}_{2}$ - une consonne qui s'est longtemps maintenue dans la norme après voyelle brève et plus longtemps encore dans les parlers du Nord-Ouest. (Elle était prononcée dans la langue décrite par La Touche, mais effacée dans celle de Vaudelin).

Comment se fait-il que les reflets des toniques de -ĀTōs, -ĪTōs, -ŪTōs de courbés, finis et nus du paradigme $\mathrm{A}_{1}$ ne soient pas longs en gallo? Les reflets préconsonantiques de ces participes passés auraient pu se généraliser à la pause, comme l'a fait le reflet préconsonantique de la désinence de $2 \mathrm{pl}-e z<-\bar{A} T$ TIS xii , qui est normalement une voyelle longue ou dont le timbre a les caractéristiques des anciens [e:] longs.

Il est vraisemblable, comme le propose Chauveau, que la tonique brève des pluriels du paradigme $A_{1}$ est le reflet phonétique des formes prépausales du XVI $\mathrm{XI}^{\mathrm{e}}$ siècle dont le [s] final a fini par s'effacer dans cette position sans provoquer d'allongement compensatoire. Il a donc dû exister une période pendant laquelle il existait deux formes en compétition pour le pluriel des paradigmes $\mathrm{A}_{1}$ et $\mathrm{A}_{2}$, toutes les deux sans $s$ final : l'une avec une tonique brève, l'autre avec une tonique longue. C'est la première qui s'est généralisée pour le paradigme $\mathrm{A}_{1}$ et la seconde pour le paradigme $\mathrm{A}_{2}$. Comment rendre compte de cette différence?

Plusieurs pistes se présentent que je ne pourrais pas développer ici : (1) plus grande fréquence des pluriels du paradigme $A_{1}$ en position prépausale, (2) conflit homonymique de la forme au pluriel avec la forme du féminin, (3) association entre la durée au pluriel et la présence d'un [t] final au singulier. Pour la seconde, on voit en effet dans le tableau 4 que la confusion entre les singuliers et les pluriels se produit lorsque le pluriel aurait été identique à la forme correspondante du féminin : le choix de la variante ayant une tonique brève au pluriel aurait ainsi évité une confusion gênante avec le féminin (on explique cependant mal la confusion des nombres lorsqu'il n'existe pas de forme du féminin correspondante). La troisième se justifierait s'il s'avérait qu'au moins une partie des anciens noms et adjectifs du paradigme $B_{1}$ avaient fini par devenir invariables.

\section{Le système classique}

Le développement du système classique dans la norme du français se devine progressivement dans les témoignages des grammairiens au cours du XVII ${ }^{\mathrm{e}}$ siècle. Ceux $\mathrm{du} \mathrm{XVI}$ siècle ne sont pas vraiment pertinents, dans la mesure où ils décrivent un état de langue où l'on prononçait les [s] postvocaliques en finale de mot. Les éléments du système classique se trouvent dans la deuxième édition en 1696 du traité de Hindret, mais n'apparaissaient pas encore dans l'édition de 1687. C'est probablement l'autorité 
d'Olivet qui l'établit définitivement dans la norme des grammairiens. Sa formulation est donnée à l'occasion d'une remarque incidente dans l'édition originale en 1736 de son traité sur la prosodie du français; ce n'est qu'après 1760 qu'on en trouvera la formulation définitive :

Il eft à remarquer, que les pluriels de tous les mots, dont la terminaiffon eft mafculine, font longs : des almanāchs, des fācs. (Olivet $1736: 59$, s. v. Ac, formulation identique en 1760 )

Une fois pour toute, faisons ici mention de cette autre règle, qui est sans exception. Toute syllabe masculine, qu'elle soit brève ou non au singulier, est toujours longue au pluriel : des sācs, des sēls, des pōts, etc. On doit même étendre cette règle jusqu'aux singuliers masculins, dont la finale est l'une des caractéristiques du pluriel : le têmps, le $n \bar{e} z$, etc. (1807: 48, s. v. Ac, reprise à la page 70 - il n'est pas sûr qu'on trouve cette addition dans les éditions publiées avant la mort de l'auteur en 1768)

L'effet du chva posttonique pour la morphologie du genre avait cependant été formulé beaucoup plus tôt :

C'eft une Régle générale, \& qui regarde également les autres voyelles, Que tous les mots qui finiflent par un E muet, immédiatement précédé d'une voyelle, ont leur penultiéme longue : penfēe, aimēe, je liē [sic], je me fiē [sic] ; jōie, j'envōie ; je lōue, il jōue; je nūe, il mūe. (Olivet 1736: 73, s. v. ÉE; l'édition de 1807 y substitue des exemples plus illustratifs pour le féminin: pensēe, armēe; je līe, je me fìe; jōie, j'envōie ; je lōue, il jōue; la nūe, la rūe)

\section{L'évolution récente de la norme parisienne}

Un ensemble d'indices permettent de croire que les oppositions de durée vocalique commencent à disparaître à la finale absolue au début du $\mathrm{XIX}^{\mathrm{e}}$ siècle, en ne laissant subsister que les distinctions de timbre $[a] \neq[a],[œ] \neq[\varnothing],[0] \neq[0]$ qui en sont issues. Sophie Dupuis (1836) qui relève la distinction $[a] \neq[a]$ à la finale absolue, comme dans bras $[\mathrm{bra}] \neq$ gras $[\mathrm{gra}]$, semble dire que les alternances attendues entre le singulier et le pluriel ont disparu, et note en particulier l'homophonie entre le singulier sopha et son pluriel sophas (1836: 8); elle ne discute cependant pas des cas où le $a$ était suivi d'une autre consonne que $s$. La distinction $[0] \neq[0]$ qui marquait les oppositions de nombre des mots comme sot, fagot, etc., disparaît de la norme des lexicographes en 1830 (cf. Morin, Langlois et Varin 1990). Toutes les distinctions de durée ont disparu à la finale absolue du dictionnaire de Michaelis et Passy (1897). Les différences de timbres $[a] \neq[a]$ et $[0] \neq[0]$ sont toujours distinctives en finale absolue ([o] en final de mot ne survivant cependant que dans l'adverbe trop), mais ne jouent aucun rôle dans la morphologie du nom.

Les observations de l'abbé Rousselot (Rousselot et Laclotte 1913) et de Marguerite Durand (1936) au début du $\mathrm{XX}^{\mathrm{e}}$ siècle montrent cependant que les anciennes distinctions de nombre n'ont pas totalement disparu à Paris et dans la région parisienne :

Les mots de cette série [-aux, -asm-ats, -ois, -eux, -eaux, -ots, -is, -its, -us, -uts, -ous, -oux] ont été modifiés par la chute de l's finale et par la distinction du singulier et du pluriel, qui en a été la conséquence. La loi du nombre est encore très vivante dans le français régional de l'ouest. Et l'on peut même en trouver des traces à Paris où bien des personnes encore, les unes consciemment, les autres leur insu, allongent les voyelles finales au pluriel. Un vieillard de la cité disait «un marmouset» avec un $e$ moyen, et «des marmousets » avec un è ouvert long; un autre distingue « un doigt » avec un $a$ moyen et « des doigts » avec un $a$ fermé.

Mais ces distinctions s'effacent, et l'unification se fait entre le singulier et le pluriel. C'est le singulier qui l'a emporté dans les mots en $-a t$, $-a$. Le pluriel triomphe dans tous les autres cas. (Rousselot et Laclotte $1913: 137-138$ )

Pas plus que les distinctions de genre :

Les voyelles suivies d'un $e$ muet final sont généralement fermées et quelques peu plus longues que si elles étaient seules. (Rousselot et Laclotte $1913: 140$, qui notent toutes ces voyelles à l'exception de [i] et [₹], d'un signe explicite de durée)

L'œ final [sc. le $e$ muet] se sent encore quelquefois dans ue $\left[\mathrm{y}^{\infty}\right]$, eue $\left[\aleph^{\infty}\right]$, [egwij tordy $\bar{y}^{\infty}$ ] «aiguilles tordues »; [pœtit fij zufly $\bar{y}^{\infty}$ ] « petite fille joufflue », [pœtit fij nȳ ${ }^{\infty}$ ] 


\begin{abstract}
«petite fille nue ». - $\left[\mathrm{rob} b l \varnothing^{\infty}\right]$ « robe bleue ». Ces formes recueillies de la bouche d'une personnage d'âge moyen et à son insu, ne se retrouvent plus chez les jeunes. Le suffixe -rie tend à prendre un $i$ moyen. Ce fait inconnu de personnes de 60 ans commence avec la génération de 1850 dans les mots les plus vulgaires (boucherie, boulangerie), tandis que les plus nobles (joaillerie) sont intacts. Chez les jeunes, la réduction paraît faite. (Rousselot et Laclotte $1913: 141$ )
\end{abstract}

Distinctions que Remacle (1994) pense pouvoir retrouver à Paris à la veille du XXI ${ }^{\mathrm{e}}$ siècle dans un essai très provocateur, que personne cependant n'a jugé utile de mettre à l'épreuve de la vérification empirique (cf. Morin 2000: 116-117).

On ne peut s'empêcher de remarquer que les distinctions de genre semblent s'être maintenues plus longtemps que celles de nombre. Ce décalage dans le temps peut très bien trouver sa source dans la dynamique des distinctions morphologiques. Les transcriptions phonétiques des terminaisons $-u e\left[\mathrm{y}^{\infty}\right]$ et eue [œ ${ }^{\infty}$ ] notées par l'abbé Rousselot, auxquelles font écho celles de Marguerite Durand (1936: 250-252) pour les terminaisons $-e ́ e ~\left[\mathrm{e}^{\curvearrowright}\right]$ et $-u e\left[\mathrm{y}^{\circ}, \mathrm{y}: \mathrm{i}\right]$ dans la région parisienne vingt ans plus tard, cependant, laissent croire que les toniques longues issues de la coalescence avec un chva posttonique ont conservé une qualité propre que n'avaient pas nécessairement les autres toniques longue - comme le relevait l'abbé Boulliette au XVIII siècle probablement dans une variété régionale du français parlé en Bourgogne (cf. note ii). Il est fort probable que le modèle classique de la norme parisienne n'était peut-être pas aussi bien partagé dans la capitale que les grammairiens ne le laissaient entendre

\title{
6 Conclusion
}

Le $-s$ de flexion a eu des effets différents sur la durée puis le timbre d'une tonique précédente selon qu'il se trouvait à la pause, en sandhi devant consonne ou en sandhi devant voyelle. Son évolution devant voyelle est à la source des liaisons modernes qui n'ont pas été examinées ici.

En finale absolue à la pause, ses effets sur une voyelle tonique précédente sont différents selon qu'il est issu d'une affriquée [ts] ou de la fricative [s] de l'ancien français primitif. Ce sont ces effets que décrivent les grammairiens du XVI ${ }^{e}$ siècle, alors que les [s] finaux étaient encore prononcés à la pause, au moins dans la langue des lettrés. Le système des oppositions de durée qui se dégage de leur description n'est que minimalement pertinent pour l'étude de l'évolution ultérieure des oppositions de nombre.

Les formes du pluriel qui s'était développées en sandhi devant consonne, où le $s$ en s'effaçant avait allongé la voyelle précédente, se généralisent à la pause à partir du XVII ${ }^{\mathrm{e}}$ siècle en remplacement des formes originales. La longueur de la tonique au pluriel caractérisera longtemps la marque du nombre des noms et adjectifs dans le système classique défendu par les grammairiens, où elle note aussi le féminin d'un grand nombre de noms et d'adjectifs, après que les chvas posttoniques aient fusionné avec une tonique précédente.

Dans certains dialectes, en particulier ceux du nord-ouest du domaine d'oïl, les anciennes toniques brèves des masculins pluriels ont pu survivre à la pause après l'effacement des $s$ issus d'un [ts] médiéval. Les dialectes de l'est de ce domaine ont aussi connu un développement distinct du système classique, qu'on connaît mal, cependant. Dans ces parlers, la longueur de la tonique joue encore un rôle important dans la morphologie du nom, mais uniquement comme marque du féminin.

La perte de la durée vocalique en fin de mot a sonné le glas des marques morphologiques qui en dépendait dans la norme parisienne. Les marques de nombre se sont effacées même lorsque des différences de timbre s'étaient substituées aux différences de durée pour les voyelles non hautes. Tandis que $[\mathrm{a}]<[\mathrm{a}:]$ s'oppose au [a] primitif en finale de mot dans des paires comme (il) bat [ba] $\neq$ bas [ba], on n'observe plus de distinction entre chat et son pluriel chats. La norme parisienne a généralisé la forme du singulier pour les terminaisons en $a$, et la forme du pluriel dans les autres cas. Ce nivellement des distinctions de nombre est responsable du remplacement de [o] ouvert par [o] fermé en finale de mot, une lente évolution qu'on a rangée, en guise d'explication, sous le vocable de «loi de position ». On comprend difficilement ce qui conditionne le choix du singulier ou du pluriel pour la forme qui a été généralisée. On se serait attendu à ce que ce soit la forme du singulier, la plus fréquente, qui l'ait été. La même tendance s'observe d'ailleurs dans les parlers du sud de la Bretagne romane où "c'est généralement la forme du pluriel qu’on emploie pour le singulier et le pluriel » (Chauveau 1984: 162). 


\section{Références bibliographiques}

Boulliette, abbé (1788a) Traité des sons de la langue françoise et des caractères qui les représentent, nouvelle édition retravaillée et considérablement augmentée. Paris : Varin.

Boulliette, abbé. (1788b). Syllabaire françois, à l'usage de ceux qui commencent à apprendre à lire. Paris: Varin.

Bourciez, É. (1958) Précis historique de phonétique française, $9^{\mathrm{e}}$ éd. revue par les soins de Jean Bourciez. Paris : Klincksieck.

Bourciez, É. et J. (1967) Phonétique française. Paris : Klincksieck.

Chauveau, J.-P. (1984) Le gallo: une présentation. Studi 26 et 27. Brest : Université de Bretagne.

Chauveau, J.-P. (1989) Évolutions phonétiques en gallo. Paris : CNRS.

Dottin, G. et J. Langouët (1901) Glossaire du parler de Pléchâtel (canton de Bain, Ille-et-Vilaine). Rennes : Plihon et Hommay.

Dumas, D. (1986) Le statut des « deux a » en français québécois. Revue Québécoise de Linguistique 15:2, 167-197.

Dupuis, S. (1836) Traité de prononciation ou nouvelle prosodie française. Paris : Hachette.

Durand, M. (1936) Le genre grammatical en français parlé à Paris et dans la région parisienne. Paris: Bibliothèque $\mathrm{du}$ « français moderne ».

Fouché, P. (1966) Phonétique historique du français, vol. 3: les consonnes, $2^{\mathrm{e}}$ éd.. Paris : Klincksieck.

Fouché, P. (1969) Phonétique historique du français, vol. 2: les voyelles, $2^{\mathrm{e}}$ éd.. Paris : Klincksieck.

Hindret, J. (1687) L'art de bien prononcer et de bien parler la langue françoise. Paris: Laurent d'Houry.

Hindret, J. (1696) L'art de bien prononcer parfaitement la langue françoise, $2^{\mathrm{e}}$ édition revue et corrigée, 2 vol. Paris: Laurent d'Houry.

Joos, M. (1952) The medieval sibilants. Language 28, 222-231.

La Noue, O. de (1596) Dictionnaire des rimes françoises. Genève : les héritiers d'Eustache Vignon.

La Touche, P. de (1696) L'Art de bien parler françois. Amsterdam : H. Desbordes.

La Touche, P. (1730) L'art de bien parler françois, qui comprend tout ce qui regarde la grammaire, \& les façons de parler douteuses, 4 éd. Amsterdam : Wetsteins \& Smith.

Martinet, A. (1955) Économie des changements phonétiques - Traité des changements phonétiques. Berne: Francke.

Michaelis, H. et P. Passy (1897) Dictionnaire phonétique de la langue française. Hanover/Berlin : Carl Meyer.

Morin, Y. Ch. (1999) La graphie de Jean-Antoine de Baïf: au service du mètre! L'écriture du français à la Renaissance - Orthographe, ponctuation, systèmes scripturaires. Nouvelle Revue du Seizième siècle 17/1, 85106.

Morin, Y. Ch. (2000 [2001]) Le français de référence et les normes de prononciation. Le français de référence. Constructions et appropriations d'un concept, éd. par Michel Francard, Geneviève Geron et Régine Wilmet. Actes du colloque de Louvain-la-Neuve (3-5 novembre 1999). Volume I : Cahiers de l'Institut de Linguistique de Louvain 26, 91-135.

Morin, Y. Ch. (2003) Syncope, apocope, diphtongaison et palatalisation en galloroman: problèmes de chronologie relative. Actas del XXIII Congreso internacional de lingüistica y filología románica (Salamanca, 24-30 septiembre 2001), éd. par Fernando Sánchez Miret, 113-169. Tübingen : Niemeyer.

Morin, Y. Ch. (2004) Peletier du Mans et les normes de prononciation de la durée vocalique au XVI siècle. Les normes du dire au XVIe siècle, éd. par Jean-Claude Arnould et Gérard Milhe Poutingon, 421-434. Paris : Champion.

Morin, Y. Ch. (2005a) La liaison relève-t-elle d'une tendance à éviter les hiatus ? Réflexions sur son évolution historique. La liaison: de la phonologie à la cognition, éd. par Jean-Pierre Chevrot, Michel Fayol et Bernard Laks, Langages 158, 8-23. 
Morin, Y. Ch. (2005b) La graphie de Peletier. Euvres poetiques intitulez louanges aveq quelques autres ecriz. Euvres complètes de Jacques Peletier du Mans, édition critique par Sophie Arnaud, Stephen Bamforth et Jan Miernowski, sous la direction d'Isabelle Pantin, 57-67. Paris : Honoré Champion.

Morin, Y. Ch. et L. Dagenais (1988) Les normes subjectives du français et les français régionaux: la longueur vocalique depuis le $\mathrm{XVI}^{\mathrm{e}}$ siècle. Distributions spatiales et temporelles, constellations des manuscrits. Études de variation linguistique offertes à Anthonij Dees à l'occasion de son $60^{e}$ anniversaire, éd. par Karin van ReenenStein et Pieter van Reenen, 153-162. Amsterdam: John Benjamins.

Morin, Y. Ch. et G. Desaulniers (1991) La longueur vocalique dans la morphologie du pluriel dans le français de la fin du XVI ${ }^{\mathrm{e}}$ siècle d'après le témoignage de Lanoue. Actes du XVIIIe Congrès international de linguistique et de philologie romanes (Université de Trèves 1986), tome III, éd. par Dieter Kremer, 211-221. Tübingen : Niemeyer.

Morin, Y. Ch., M.-C. Langlois et M.-È. Varin (1990) Tensing of word-final [o] to [o] in French: the phonologization of a morphophonological rule. Romance Philology 43, 507-528.

Olivet, P., abbé d' (1736) Traité de la prosodie françoise. Paris : Gandouin.

Olivet, P., abbé d' (1807) Remarques sur la langue française par M. l'abbé d'Olivet, revue, et augmentée de deux lettres de l'Abbé Le Batteux, sur l'accent prosodique et sur l'accent oratoire. Paris: Barbou.

Pope, M. K. (1952) From Latin to modern French with especial consideration of Anglo-Norman, $2{ }^{\mathrm{e}}$ éd. Manchester : Manchester University Press.

Rabiet, abbé E. 1887-1890. Le patois de Bourberain (Côte-d'Or). Revue des patois gallo-romans 1, 241-255, 2, 48$53,181-186,257-269,3,27-47,88-102,161-185,142-169$ et $4,35-39,241-255$.

Remacle, L. (1994) Orthoépie - Essai de contrôle de trois dictionnaires de prononciation française. Genève : Droz.

Roche, J. B. (1777) Entretiens sur l'orthographe françoise et autres objets analogues. Nantes : Veuve Brun.

Rousselot, abbé P. et F. Laclotte. 1913. Précis de prononciation française, $2^{\mathrm{e}}$ éd. Paris : Didier / Paris-Leipzig: Welter.

Shipman, G. R. (1953) The vowel phonemes of Meigret. Georgetwon University Press : Washington, D.C.

Thurot, Ch. (1881-1883) De la prononciation française depuis le commencement du XVI siècle, d'après le témoignage des grammairiens, en 3 vol. Paris: Imprimerie Nationale.

Troubetzkoy, N. S. (1970) Principes de phonologie, traduit par J. Cantineau. Paris : Klincksieck.

Vaudelin, G. (1713) Nouvelle manière d'écrire comme on parle en France. Paris: Veuve Jean Cot et Jean-Baptiste Lamesle.

Vaudelin, G. (1715) Instructions cretiennes mises en ortographe naturelle pour faciliter au peuple la lecture de la sience du salut. Paris: Jean-Baptiste Lamesle.

Walter, H. (1993) L'évolution phonologique du français depuis le XVIII ${ }^{\mathrm{e}}$ siècle. Sprachwandel und Sprachgeschichte : Festschrift für Helmut Lüdtke zum 65. Geburtstag, éd. par Jürgen Schmidt-Radefeldt et Andreas Harder, 211220. Tübingen : Gunter Narr.

\footnotetext{
${ }^{\mathrm{i}}$ Cette recherche a été subventionnée en partie par le Conseil de Recherches en Sciences Humaines du Canada (Les voies du français, projet GTRC 412-2004-1002, sous la direction de Mme France Martineau).

ii Dans sa description du français, l'abbé Boulliette (1788a: 20-21), né en Bourgogne (cf. Thurot 1881: LXXXII), fait état de différences phonétiques importantes entre les toniques longues selon qu'elles doivent leur durée à la coalescence avec un chva post-tonique ou à l'effacement d'un $s$. Les différences qu'il décrit font penser aux distinctions connues sous le nom de "coupe syllabique" (cf. Troubetzkoy 1970: 209-210, qui cependant les observe dans des contextes différents). Les premières auraient une coupe "lente" (ou "tendre") : le « $e$ muet, feul après une voyelle à la fin des mots, ne devroit point être compté pour une fyllabe diftinguée de celle qui le précède, puifqu'il n'eft que la prolongation de cette fyllabe, \& qu'il n'eft que le refte de la même voix, mais traînée ", comme dans les mots «donnée, j'agrèe [sic], la vie, je loue, ils tuent, ils envoient, la joie». Les seconds s'observent dans les mots «du grès, je vis, des loups, je fus, des envois » et ont une coupe "brusque" : "quoique les dernières fyllabes de ces derniers mots foient très-longues, la voix n'eft point traînée, \& elle s'arrête tout court lorfque la fyllabe eft prononcée. \ Cette différence phonétique entre les deux types de longues, si elle était caractéristique des parlers de l'Est, pourrait expliquer l'évolution particulière du système de flexion nominale dans cette région.
} 
Il est certainement significatif que l'abbé Boulliette illustre les deux types de coupe pour les voyelles [E:, i:, ur, y:] et la diphtongue [u:c], mais qu'il ne donne aucun exemple de [e:] brusque en contrepartie du [e:] tendre de donnée. Quelques pages plus tôt, le savant abbé écrivait «on ne peut prononcer [...] le nez que comme il eft né »; on ne peut exclure cependant que dans le contexte où ceci est écrit, l'identité des prononciations concerne exclusivement le « fon » (c'est-à-dire du timbre) des voyelles et non leur durée. À toute fin pratique, le [e:] long brusque ne pouvait apparaître que dans le mot $n e z$, qui ne vient pas nécessairement à l'esprit lorsqu'il faut formuler des généralisations. Il n'est pas rare que nez soit ainsi “oublié". L'étude de l'abbé Rabiet sur le parler de Bourberain, par exemple, omet toute discussion sur l'évolution ce mot. Sa prononciation apparaît seulement dans les transcriptions phonétiques d'un conte populaire et de dictons $(1890: 185,254,255)$, où il est noté avec un [e:] long caractéristique, différent du [E] ouvert bref attendu devant [ts] dans ce contexte (cf. les blés, (ils sont) lassés, attablés $1890: 244,247,249$ ). On note cependant un [e:] long dans les formes de assez (1890: 178), dont la durée peut s'expliquer par sa fonction syntaxique de modificateur qui entraîne la généralisation de la variante préconsonantique, et un [e] bref pour la terminaison $-e z$ de la 2pl. (1890: 168-169), qui pourrait être la généralisation du reflet de -ĀTǏs après une consonne palatale.

${ }^{i i i}$ Fouché écrit : «La chute de $s$ final a eu pour conséquence l'allongement de la voyelle précédente. Cet allongement est encore de règle au XVI $\mathrm{XI}^{\mathrm{e}}$ siècle, et il continuera de l'être longtemps après. C'est ainsi qu'au pluriel, Domergue (1805) sera le premier à faire des restrictions à l'usage antérieur qui voulait qu'on prononçât une voyelle longue, par opposition avec le singulier » (Fouché 1969: 390). On trouve cependant quelques pages plus tôt : « il est probable que dans la langue courante l'abrègement des voyelles longues à la finale absolue [y compris les pluriels des substantifs oxytoniques] est beaucoup plus ancienne [sic] qu'ils [les grammairiens] ne le laissent entendre. Il n'est pas exagéré de le reporter jusqu'au milieu du $\mathrm{XV}^{\mathrm{e}}$ siècle, sinon plus tôt » (ibid. p. 243).

${ }^{\text {iv }}$ Depuis le $\mathrm{XVI}^{\mathrm{e}}$ siècle, les systèmes orthographiques du français obéissent à un principe d'invariance idéographique, qui privilégie l'utilisation d'une forme graphique unique du mot, indépendante des ajustements en sandhi - sauf pour les enclitiques monosyllabiques comme le, la, de, que, te, me, se, si. Dans les orthographes réformées de type phonétique, c'est la prononciation du mot à la pause qui sert de modèle à cette graphie unique. Seuls Meigret (15481551 ) et Ramus (1562) admettent des graphies spécifiques, dans des cas relativement limités, pour noter certains types de sandhi, ce que Peletier reproche notamment au premier (cf. Morin 1999).

${ }^{v}$ Meigret ne dit cependant pas que les consonnes sont muettes devant le $-s$ de flexion de lacs, cocs, hanaps, mais seulement que ces consonnes ont une « lejiere pronõçíaçío ». Il fait remarquer dans son Tretté $\left(\mathrm{p}\right.$. $17 \mathrm{v}^{\circ}-$

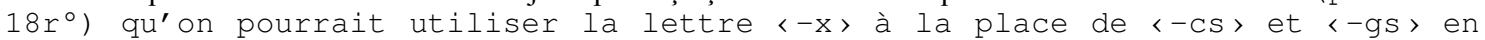
faisant attention cependant de ne pas lui donner autant de force qu'on le fait à l'initiale des nom grecs Xerxes, Xãtipe, Xenophon: la lettre 〈X〉 s'observe "ao' pluriers dę' noms tẹrminez ęn $c$, ou $g$, come lac, long, lacs, longs: més pour aotant qe nou' prononçons fort $x$, e qe cees aotres ne demãdet pas vne si viue prononçíaçíon, nous vsons plutót de son eqiuallant: cõbien q'aosi bon seroęt lax, ę lonx: qe lacs, ę longs. » Il est probable que le son [k] pouvait se prononcer dans certains registres formels sur lequel Meigret se fonde pour sa graphie. On retrouve ces prononciations formelles dans d'autres traités à cette époque. Ce genre de restauration est d'ailleurs attesté dans quelques parlers francoprovençaux du $\mathrm{XX}^{\mathrm{e}}$ siècle ayant conservé le $s$ en finale de mot.

vi On corrigera dans ce travail les transcriptions phonétiques incohérentes du $o$ ouvert en remplaçant partout le symbole [o] par le symbole [0].

${ }^{v i i}$ Ce traitement exceptionnel n'est pas rare pour les terminaisons -as : les mots coutelas, brouillas, fatras, frimas, lacs (et entrelacs), pourchas, soulas ont une tonique longue, à côté de bras, haras et materas 'matelas' avec une tonique brève. Sinon, petits, courroux (avec une tonique longue) et jolis, épis (avec une tonique brève) semblent être les seules autres exceptions.

viii Les auteurs des traités grammaticaux présentent souvent des règles de prononciation générale qui s'appuient sur des régularités graphophoniques supposées. On doit toujours prendre ces généralisations avec beaucoup de réserve. Seuls les exemples explicites sont vraiment représentatifs.

${ }^{\text {ix }}$ Roche fait de nombreuses observations sur la durée dans ses dialogues (pp. 17-103) qui seraient difficiles à synthétiser sans le tableau récapitulatif final (pp. 104-109) où il apparaît que les voyelles [i, y, u, E. ø, o, a] ne connaissent que deux degrés de durée; seul [e] en connaîtrait trois. Ce système est très différent de celui de Boulliette (cf. note ii) qui distingue trois types de durée en finale de mot pour toutes les voyelles à l'exception de $o$ et $a$ (qui n'en connaissent que deux) et pour lesquelles il peut utiliser les termes «bref, long, très long » ou "bref, long, prolongé » dans ses ouvrages didactiques (Boulliette 1788b: 17, 18, etc.).

${ }^{x}$ Chauveau (1984: 167) observe aussi qu'en gallo «les adjectifs ne distinguent pas le singulier du pluriel en général », une formulation qui inclut les participes passés en fonction d'adjectif.

${ }^{x i}$ Les pluriels de [py] 'puits' et [pə] 'pis' ne sont pas précisés ; il est vraisemblable que [pə] 'pis' est invariable ; on peut penser sinon que les auteurs auraient précisé le pluriel. Le continuateur de [ts] médiéval est devenu $[\mathrm{r}]$ après la 
voyelle brève dans [vir] 'vis', comme cela est fréquent en gallo. Le nom lacs était déjà passé au paradigme $\mathrm{C}_{2}$ dans la langue de Lanoue au XVI ${ }^{\mathrm{e}}$ siècle.

xii Si l'on tient compte des sandhis, il n'est nul besoin de postuler un traitement particulier comme dans Chauveau (1989: 36-37). 